\title{
Editorial: Was heißt Krise des Marxismus?
}

\section{Zur Konzeption dieses Heftes}

\section{Die Notwendigkeit einer marxistischen Grundsatzdebatte}

Als L. Althusser auf dem von „,ll Manifesto ${ }^{6}$ während der Biennale in Venedig im November 1977 veranstalteten Kongreß über „Macht und Opposition in den nachrevolutionären Gesellschaften“ den endlichen Ausbruch der Krise des Marxismus verkündete*, fand diese Provokation im Kontext deutlich umrissener Strategieprobleme der eurokommunistischen Parteien Italiens, Spaniens und Frankreichs statt. Inzwischen hat diese Provokation in der gesamten europäischen Linken eingeschlagen, aber damit zugleich eine eigentümliche Transformation erfahren. Gerade hierzulande wirkte sie als Signal, die subjektiven Probleme als Marxist zu artikulieren und in vielfältigsten Formen den Marxismus als handlungsorientierende emanzipatorische Theorie in Frage zu stellen. Umgekehrt forderte dies viele orthodoxe Marxisten heraus, eine Krise des Marxismus als Problem überhaupt zu leugnen, um stattdessen die marxistische Weiterarbeit in abgesteckten Bahnen umso emsiger zu propagieren. Die Folge ist, daß die Frage: gibt es eine Krise des Marxismus? gleichsam zur Gewissensfrage nach der Wahrhaftigkeit der Marxisten umgemünzt wird. Das gleicht dann mehr einem Glaubenskrieg als einer politisch-wissenschaftlich geführten Debatte um das Marxismusverständnis und die darin eingeschlossenen praktischen und theoretischen Fragen. Denn darum handelt es sich ja bei der aufgebrochenen Krise des Marxismus: Zweifel an überkommenen Selbstverständlichkeiten, an politischen Identifikationen und an gewohnten theoretischen Begründungszusammenhängen. Will man nicht lediglich subjektiver Verzweiflung anheimfallen, kann es nur sinnvoll sein, den Zweifeln auf den Grund zu gehen, d.h. präzise dạrüber zu diskutieren, welche Elemente im Marxismus problematisch geworden sind und an welchen begründet festgehalten werden kann.

In diesem Sinne einer zu führenden Grundsatzdebatte über den Marxismus kann die Titelfrage: was heißt Krise des Marxismus? nicht ex cathedra definiert und womöglich noch beantwortet werden. Vielmehr müssen zunächst einmal alle Dimensionen, die in der Krise des Marxismus aufgebrochen sind, ohne sektiererische $\mathrm{Ab}$ grenzungen ernst genommen, präzisiert und diskutiert werden, in der Hoffnung, daß aus einem solchen Diskurs wirklich neue und richtungsweisende Antworten entste-

* Die in den einzelnen Thesenpapieren des öfteren angesprochene Diskussion während der Biennale in Venedig im November 1977 wird demnächst im Verlag ,Ästhetik und Kommunikation" dokumentiert: Elmar Altvater, Otto Kallscheuer (Hrsg.), Den Staat diskutieren, erscheint ca. Oktober $1979(a ̈+\mathrm{k}$, Berlin West) 
hen. Dieses Ziel, zur Strukturierung der Marxismusdiskussion beizutragen, hat sich die PROKLA mit der Konzeption dieses Heftes gesetzt und soll auch in den folgenden Heften weiterverfolgt werden.

\section{Die Struktur des Heftes}

Den Auftakt bildet ein Diskussionsforum, in dem kürzere Thesenpapiere von L. Magri, F. O.Wolf, B. Blanke / G. Schäfer, W. Spohn und P. Oehlke zu einzelnen Aspekten der Marxismusdiskussion abgedruckt sind. Ein ausführlicherer Beitrag von A. Mohl schließt sich an. Mit dem Referat von L. Magri über die ,Krise des Kapitalismus und Krise der Linken ' und der Skizze der Position Althussers durch F. O. Wolf: ,Auflösung oder Emeuerung des Marxismus' soll zunächst der politisch-theoretische Ausgangspunkt der Debatte um die Krise des Marxismus, nämlich die Strategie- und Theorieprobleme des Eurokommunismus, umrissen werden. Der Diskussionsbeitrag von $B$. Blanke und $G$. Schäfer: Krise der Linken - Krise des Marxismus'versucht dann, diese Debatte in ihrer Relevanz für die bundesrepublikanische Linke deutlich zu machen. In den Thesenpapieren von $W$. Spohn und $P$. Oehlke wird der historische Kontext der Marxismusdebatte angerissen. W. Spohn versucht dabei in seinen ,Thesen zum historischen Verständnis von Marxismus und Arbeiterbewegung' diesen historischen Kontext von der Geschichte der Arbeiterbewegung her zu thematisieren. Der Diskussionsbeitrag von P. Oehlke:, Probleme der Marxismusdiskussion in der Bundesrepublik' legt in Kritik an den Spohnschen Thesen den Akzent auf die bewahrenswerten Teile der marxistisch-leninistischen Tradition. Schließlich wird von $A . M o h l$ mit ihrem Aufsatz: ,Was ist wissenschaftlicher Sozialismus"' in der Kritik am objektivistischen Kapitalverständnis, das nicht nur für den Marxismus der 2. und 3. Internationale, sondern in vielen Elementen auch für den Neomarxismus der Neuen Linken prägend war, eine sozialemanzipatorische Lesart der Marxschen Theorie ent wickelt. Auch die weiteren Beiträge in diesem Heft fügen sich in die Diskussion um die Krise des Marxismus ein. Chr. Neusüß fragt in ihrem Beitrag nach den neuen gesellschaftlichen Momenten in der gegenwärtigen ökonomischen Stagnationsphase, die die aktuelle Krise von den vergangenen kapitalistischen Krisen unterscheiden und die neue Antworten vonseiten einer marxistischen Theorie und Politik verlangen. U. Jürgens nimmt die Diskussion um das zentrale Gesetz der Marxschen Kapitalanalyse, das „Gesetz des tendenziellen Falls der Profitrate“, auf und versucht, den dieses Gesetz konstituierenden Widerspruch auf der Ebene des Einzelkapitals als handlungsrelevant aufzuspüren, indem er die übergangstheoretischen Implikationen des Gesetzes (z. B. Aktiengesellschaften) und die Antizipationsstrategien gegen die Wirkungen des Gesetzes im Rahmen der betriebswirtschaftlichen Diskussion analysiert. Und schließlich untersucht $R$. Dombois auf Basis eigener und fremder empirischer Studien zum Arbeiterbewußtsein in der Krise jene Mechanismen, die auf der Betriebsebene die (z. B. in deterministischen Theorien unterstellten) Politisierung durch die Erfahrung der Krise blockieren - dabei schließt dieser Artikel an die in PROKLA 19/21 und 26 veröffentlichten Thesen zur Krisenerfahrung/verarbeitung an. 
Mit diesen Beiträgen wollen wir relevante Aspekte der Diskussion um die Krise des Marxismus ansprechen, aber damit sind doch nicht alle Dimensionen dieser Debatte eingeholt, geschweige denn in der materialen Analyse weitergeführt. Aus diesem Grunde sollen die Diskussionsebenen inhaltilch vorweg umrissen werden.

\section{Die Krisen des Marxismus \\ 1. Eurokommunismus}

Um nicht ins vage Rumdeuteln über die Krise des Marxismus zu geraten, ist es unumgänglich, sich den Ausgangspunkt dieser Diskussion im Kontext der eurokommunistischen Arbeiterbewegung klar vor Augen zu halten. (Vgl. hierzu L. Magri und F. O. Wolf.) Es handelt sich um eine spezifische Tradition der Arbeiterbewegung, die - im Rahmen der Kommunistischen lnternationale entstanden und nach deren Auflösung noch lange im Schlepptau Moskaus - sich unter dem Druck der entstehenden Massenbewegungen insbesondere seit Ende der 60er Jahre im praktischen und theoretischen Loslösungsprozeß vom Marxismus-Leninismus Stalinscher Prägung befindet. Wie unterschiedlich und ambivalent dieser Loslösungsprozeß in Italien, Spanien und Frankreich auch immer vonstatten gehen mag, so sind doch zentrale strategische Maximen in Zweifel geraten und verändert worden. Die selbstverständliche Orientierung auf die Sowjetunion als dem Modell des Sozialismus ist problematisch geworden, nicht nur aufgrund des sowjetisch-chinesischen Bruchs, sondern vor allem aufgrund der internen und externen Gewaltpolitik des sowjetischen Staates (Einmarsch in die Tschechoslowakei, Unterdrückung oppositioneller Dissidenten). Dies schlug sich in der Aufgabe der Formel der Diktatur des Proletariats nieder und in der Betonung eines eigenständigen Wegs zum Sozialismus. Damit war aber nicht nur die Diskussion über das Gesellschaftssystem der Sowjetunion, sondern über das eigene sozialistische Ziel freigegeben. Wie vage auch immer die neue Zielbestimmung der Verbindung von Demokratie und Sozialismus sein mag, sie mußte auch die Entwicklung zum Sozialismus hin, damit das Verhältnis zur bürgerlichen Demokratie und das Verhältnis von Partei und Klasse vorn der Bündnisfrage bis zur internen Parteistruktur betreffen. Diese Neuorientierung in der Politik der kommunistischen Arbeiterbewegung ist nun aber nicht einfach Folge der Entwicklung der Sowjetunion - der Höhepunkt des stalinistischen Terrors war ja schließlich vorüber - , sondern primär Resultat der internen Dynamik der Klassenkämpfe und sozialen Bewegungen im eigenen Land. Nun ist allerdings diese soziale Klassenbasis keineswegs homogen. Einerseits handelt es sich um eine erweiterte und sich in Wählerstimmen ausdrückende Volksopposition, andererseits um ziemlich heterogene Formen sozialer Dynamik. Nicht nur die vielfältigen Formen der neuen sozialen Bewegungen: Studenten-, Frauen-, Ökologie- und sonstige Alternativbewegungen im sogenannten Reproduktionsbereich meldeten in ungekanntem Maß ihre emanzipatorischen Ansprüche an, sondern auch in der Arbeiterklasse im Produktionsbereich, gerade auch in den Gewerkschaften entstanden ganz neue Formen sozialer Auseinandersetzungen, die sich nicht nur höhere Löhne und bessere sozialstaatliche Absicherung, sondern 
auf die qualitative Veränderung des Produktionsprozesses und der Betriebsorganisa. tion richteten und mit neuen politischen Formen verbunden waren. Dieser veränderte klassenpolitische Kontext stellt den praktischen lnhalt der Krise des Marxismus dar, der in seinen sowjetmarxistischen Traditionsmustern angesichts der neuen sozialen Tendenzen in seinem Selbstverständnis der Konstitution des proletarischen Klassenkampfs zentral getroffen wurde.

\section{Neue Linke in der Bundesrepublik.}

Wenn nun in der Bundesrepublik die Krise des Marxismus von der Neuen Linken sehr rasch aufgegriffen wurde, so traf sie hier zunächst einmal auf ein ganz anderes klassenpolitisches Milieu und entsprechend andersartig wurde auch die Debatte um die Krise des Marxismus geführt. (Vgl. hierzu die Thesen von B. Blanke und G. Schäfer.) Während einerseits die sowjetmarxistische Tradition in der DKP unbeirrt linientreu mit den Folgen politischer Bedeutungslosigkeit bewahrt wurde, und andererseits die Masse der Arbeiterklasse weitgehend gewerkschaftlich und sozialdemokratisch korporativistisch integriert war, blieb die Entwicklung des Marxismus wesentlich Produkt der politisch isolierten Studentenbewegung. Der Marxismus war vornehmlich Intellektuellenmarxismus, die Krise des Marxismus wurde entsprechend vornehmlich als Theoriekrise des Marxismus erfahren. Trotz dieses zentralen nationalen Unterschieds hinsichtlich der Existenz einer marxistisch orientierten Massenbewegung der Arbeiterklasse reflektiert diese Theoriekrise die gleichen praktischen Inhalte. Symptomatisch hierfür war das breite Interesse an der Auseinandersetzung mit der Alternative Rudolf Bahros. Die Ablehnung des realen Sozialismus als gesellschaftsemanzipatorische Perspektive, die Aufhebung der alten Arbeitsteilung auf Basis der modernen Produktivkräfte, die Humanisierung der Technologie, die $\mathrm{Zu}$ rücknahme des Staats in eine neue Form sozialer Assoziation, die reich entfaltete Persönlichkeit als Planungsziel, die Herstellung wirklich sozialer Gleichheit zwischen Mann und Frau, die Vergesellschaftung der Erziehung und im Interesse dieser emanzipatorischen Alternative ein neuer kommunistischer Parteitypus - alles Themen, die, wenn auch aus DDR-Sicht geschrieben, im Grunde die neuen sozialen Tendenzen auch in Westeuropa aufgreifen. Für die Neue Linke blieb dies vornehmlich ein theoretisches Aufgreifen. Die vorherrschende Isolation des Neomarxismus - verstärkt durch seine Akademisierung - nicht nur gegenüber der Arbeiterklasse, Gewerkschaften und Sozialdemokratie, sondern gerade auch gegenïber den neuen sozialen Bewegungen, die in vielen Ausläufern an die neu ge schaffene Massenuniversität brandeten, beförderte die Tendenz zur privatisierten Verarbeitung und Rationalisierung. Die Krise des Marxismus schien unversehens aus einer strategischen Umorientierung einer spezifischen Tradition des kommunistischen Marxismus in eine existentielle Krise vieler Marxisten verwandelt. Mag sie auch korrespondieren mit der allenthalben beobachtbaren neuen Innerlichkeit, muß sie doch als spezifisch gesellschaftliches Phänomen begriffen werden, das seinen Grund nicht nur in den vorherrschenden ,entsubjektivierten ${ }^{6}$ Marxismustraditionen hat, sondern in der gesell- 
schaftlichen Abspaltung und Unterdrückung emanzipatorischer Tendenzen hat. Aber die Privatisierung gesellschaftlicher Probleme macht nicht den Inhalt der Krise des Marxismus aus.

\section{Geschichte der Krise des Marxismus}

Wie unterschiedlich nun immer das klassenpolitische Milieu in den einzelnen Nationalstaaten Westeuropas sich darbietet und wie unterschiedlich entsprechend die Diskussions- und Verarbeitungsformen der Krise des Marxismus aussehen - der allgemeine Kern der gegenwärtigen Krise des Marxismus besteht darin, daß im Kontext der gegenwärtigen Wirtschaftkrise die Dynamiken sozialer Bewegungen, und zwar im europäischen Maßstab, anders verlaufen, als es der traditionelle Parteimarxismus und viele an ihm partizipierende Marxismusströmungen erwartet haben. Damit wird die Frage aufgeworfen, inwieweit der Marxismus bzw. die verschiedenen marxistischen Strömungen den eigenen Anspruch, gesellschaftsemanzipatorische Theorie und Handlungsorientierung zu sein, erfüllt oder nicht erfüllt.

Diese allgemeine Fragestellung nach dem Verhältnis von Marxismus und gesellschaftlicher Emanzipation wirft auch ein neues Licht auf die historische Dimension der Krise des Marxismus. Wenn um die Jahrhundertwende in der Sozialdemokratie zum erstenmal über die Krise des Marxismus diskutiert wurde, so stand in Frage, inwieweit die in der Tat so erfolgreiche sozialdemokratische Parteipraxis mit ihrer reformpolitischen und demokratischen Realpolitik nicht eher die Integration in den bürgerlichen Staat betrieb. Und diese Frage wurde gestellt, weil die Konstitutionsformen des Klassenkampfs in Europa - in den sich radikalisie renden Streiks, in den politischen Widerstandsbewegungen und den revolutionären Zuspitzungen den strategischen Maximen der Sozialdemokratie zuwiderliefen und deshalb auch den Parteimarxismus der 2. Internationale problematisierten. Diese Risse zwischen Klassenbasis und Partei machten sich dann auch schlagend bemerkbar in Spaltungen - so in Rußland der Bruch zwischen Bolschewiki und Menschewiki - und in Abspaltungen -- so das Verhältnis zu Anarchisten und Syndikalisten.

Wenn dann 1931 von K. Korsch zum zweitenmal die Krise des Marxismus thematisiert wird, so geschah dies auf dem klassengeschichtlichen Hintergrund, daß auch der in der Auseinandersetzung mit dem Revisionismus etablierte revolutionäre Marxismus als theoretischem Selbstverständnis der kommunistischen Bewegung noch viel dramatischeren Zerreißproben ausgesetzt war als der Marxismus der 2. Internationale. Die Konstruktion des Marxismus-Leninismus in der Sowjetunion war das ideologische Begleitspiel der Stalinisierung von Staat und Gesellschaft und der damit einhergehenden politischen Ausgrenzung aller eigenständigen sozialrevolutionären, sozialdemokratischen, oppositionell-kommunistischen und sich schließlich in der Linken Opposition zusammenfindenden Strömungen. Ganz parallel fanden die Ausgrenzungen in den westeuropäischen kommunistischen Parteien über den Einfluß der Kommunistischen Internationale statt, zementierten die Spaltung der Arbeiterbewegung und waren ein zentraler Faktor der Hilfiosigkeit gegenüber dem her- 
einbrechenden Faschismus.

Wenn nun seit 1977 zum drittenmal die Rede von der Krise des Marxismus ist, so wäre die marxismuskritische Frage zu stellen, welche historische Kontinuitäten und Diskontinuitäten zwischen diesen drei Spielarten der Krise des Marxismus bestehen. Dies ist das Thema der Diskussionsbeiträge von W. Spohn und P. Oehlke, bedarf aber viel intensiverer Analysen der Geschichte der Arbeiterbewegung. An dieser Stelle soll nicht weiter darauf eingegangen, stattdessen der Versuch gemacht werden, kurz die theoretischen und politischen Dimensionen, die durch die heutige Krise des Marxismus aufgeworfen werden, diskussionsstrategisch zu gliedern (ausführlicher hierzu der Diskussionsbeitrag von B. Blanke und G. Schäfer). Wir folgen der Einteilung L. Magris, wenn er in seinem Beitrag Ökonomismus, Etatismus und Industrialismus als Kern der Krise des Marxismus unterscheidet und werden diese Dimensionen als Objektivismus zusammenfassen.

\section{Strategische Dimensionen der Krise des Marxismus 1. Ökonomismus}

Durch die Wirtschaftskrise seit 1973, in der weltweit die lange Aufschwungphase der Kapitalakkumulation in eine Stagnationsphase umschlug, und die in ihr sich konstituierenden Formen des Klassenkampfs und der sozialen Bewegungen wurde die alte marxistische Tradition einer ökonomischen Krisen-, Klassen- und Revolutionstheorie nachhaltig in Frage gestellt. Nun zeigen die jüngsten marxistischen Wirtschafts- und Krisenanalysen viele historisch neue Momente - von der strukturellen Arbeitslosigkeit, der Stagflation, der Rückkehr zum Monetarismus, der Austeritätspolitik bis hin zu einer neuen internationalen Arbeitsteilung - im Verlauf dieser weltweiten Überakkumulationskrise (vgl. hierzu den Beitrag von C. Neusüß). Entscheidend für die Krise des Marxismus ist jedoch, daß sich die revolutionsstrategischen Erwartungen hinsichtlich der Konstitution und Radikalisierung eines proletarischen Klassenbewußtseins, die fast alle Marxismen an die kapitalistische Krise knüpften, sich keineswegs erfüllten. Was man eigentlich schon seit der Weltwirtschaftskrise 1929 und der sie begleitenden Demoralisierung und Dissoziation der Arbeiterklasse hätte wissen müssen, wurde als soziale Erfahrung, wenn auch nicht in dem Ausmaß, wiederholt: einerseits gelang kein entscheidender Durchbruch der marxistischen Arbeiterbewegung, wurde sogar durch Rechtstendenzen überboten, andererseits nahmen Demoralisierung, subkulturelle Verarbeitung und Diversifikation der sozialen Bewegungen zu. Damit war der marxistisch oft angenommene Automatismus von Krise und Revolution, die ökonomistische Zusammenbruchstheorie im Kern in Frage gestellt. Inwieweit Marx selbst mit der Rede von den Naturgesetzen der kapitalistischen Produktionsweise und ihrer Aufhebung diesen Ökonomismus geteilt hat, ist nicht eindeutig: A. Mohl versucht zu beweisen, daß Marx gerade mit seiner Kapitaltheorie nicht ein objektivistisches Revolutionskonzept, sondern ein praktisches Emanzipationsmodell verbunden habe. Unstrittig ist jedoch, daß der Marxismus der 2. Internationale aufgrund der Krisenhaftigkeit des 
Kapitalismus und der notwendigen Verelendung des Proletariats den Zusammenbruch des Kapitalismus als naturnotwendig ansah, und der Marxismus der 3. Internationale diesen Ökonomismus fortsetzte, wenngleich sich die Begründung für den Zusammenbruch des Kapitalismus von endogenen Faktoren auf die exogene Existenz der Sowjetunion und später des sozialistischen Lagers und der dadurch institutionalisierten Systemkonkurrenz verlagerte. An diesen Traditionen partizipierte mehr oder minder der Neomarxismus in der Bundesrepublik und mußte nun die Brüchigkeit dieser Traditionen aufgrund der Wirklichkeit der Wirtschaftskrise und der subjektiven Prozesse in ihr am eigenen Leibe erfahren. Die Konstitution des Klassenkampfs und -bewußtseins war als Frage praktischen Handelns und kritischer Antizipation wieder in ihr Recht gesetzt.

\section{Etatismus}

Durch die politischen Entwicklungen in Chile, Portugal, Spanien, Italien und Frankreich und die Rolle der kommunistischen Parteien in diesen Gesellschaften ist das Staatsproblem als Frage politischen Handelns in neuer Weise aktuell geworden. Fast seit einem halben Jahrhundert schien die marxistische Vorstellung vom Kampf gegen die herrschenden Klassen und die Errichtung der Diktatur des Proletariats den revolutionären Weg zum Sozialismus zu markieren. Die Sowjetunion schien diese sozialistische Entwicklung, wenngleich mit Widersprüchen und Fehlern zu vollziehen, viele relativ unterentwickelte Gesellschaften folgten nach antiimperialistischen Revolutionen diesem Modell. Nicht nur mit der Politik dieser sozialistischen Länder ihrer Übernahme kapitalistischer Mechanismen, ihrer internen Repression und ihren gegenseitigen Fein dseligkeiten - geriet in Zweifel, inwieweit die von einer kommunistischen Partei angeführte Verstaatlichung von Produktion und Gesellschaft zur Verwirklichung eines emanzipatorischen Sozialismus ausreichte. Auch und gerade die Klassendynamik in den entwickelten kapitalistischen Ländern zeigte, daß die staatssozialistische Revolutionsvorstellung nicht automatisch emanzipatorische Inhalte verbürgte. Inwieweit Marx an einer solchen Konzeption des Staatssozialismus partizipierte, kann ihm vielleicht für die Zeit der 48er Revolution unterstellt werden, die Darstellung der Pariser Commune als tendenzieller Zurücknahme des Staats in die Gesellschaft legte den Akzent keineswegs nur auf die Eroberung des Staats, sondern auf die gesellschaftlichen Veränderungen unter der politischen Form der Commune. Erst die lassalleanische Deutung der Marxschen Theorie innerhalb der Sozialdemokratie konstituierte die einflußreiche Konzeption, nach der durch die Verstaatlichung der Großkonzerne der friedliche Übergang zum Sozialismus möglich sei. Diese Sozialismuskonzeption bestimmte auch die Revolutions" und Transformationstheorie der Bolschewiki und des durch sie geprägten Marxismus der 3. Internationale, nur daß der friedliche Übergang und die bürgerliche Demokratieform ad acta gelegt wurden. Die bitteren Erfahrungen mit dem Stalinismus und mit dem Faschismus bewiesen überdeutlich, daß die bürgerliche Demokratie material in die sozialistische Demokratie eingehen muß und nicht einfach liquidiert werden kann. Diese Konse- 
quenz wurde dann auch nach und nach vom westeuropäischen Kommunismus gezogen. Dies war auch der geschichtliche Hintergrund der neomarxistischen Staatsdiskussion in der Bundesrepublik. Allerdings stand im Vordergrund des ErkenntnisInteresses, daß der heutige Wohlfahrtsstaat nach wie vor ein bürgerlicher Klassenstaat sei und mündete in einer weitverzweigten Form- und Tätigkeitsanalyse des bürgerlichen Staats, die im wesentlichen dessen Grenzen im perspektivischen Vergleich zu sozialistischen Gesellschaftsverhältnissen beweisen sollte. Doch blieb dies insgesamt eine destruktive Analyserichtung, an der der Rekurs auf Gramsci höchstens die ökonomistische Reduktion des Politischen beseitigte. Mit der Notwendigkeit konkreten politischen Verhaltens angesichts staatlicher Repressionen ( Berufsverbote, Antiterrorgesetzgebung, Unvereinbarkeiserklärungen etc.) wurde allerdings offenkundig, wie wenig die Staatsdiskussion zur polititschen Orientierung verholfen hatte. Die Beantwortung der Frage, wie im bürgerlichen Staat zu agieren und dieser in emanzipatorischer Perspektive aufzuheben sei, steht deshalb als praktisches wie theoretisches Problem nach wie vor im Zentrum marxistischer Staatsanalyse.

\section{Industrialismus}

Durch das hypertrophe Wachstum der kapitalistischen Produktivkräfte - das weltweit sehr Wenigen Überfluß beschert und die Probleme der Armut verschärft, das die Natur als Grundlage der Produktion zu vernichten wie den Menschen und dessen Reichtum als Ziel der Produktion zu verschlingen droht - entstanden in historisch nie gekanntem Umfang soziale Bewegungen, die an den sichtbarsten Katastrophen ihren Widerstand artikulierten und formierten. Ökologie- und Antikernkraftbewegungen, Bürgerinitiativen in den Städten und Alternativbewegungen auf dem Land richten sich direkt gegen die kapitalistische Form der materiellen Produktion der Lebensbedingungen. Damit tritt zum erstenmal in der Geschichte massenhaft die Notwendigkeit einer anderen Form der Technik, d. h. eines anderen Verhältnissis von Mensch und Natur, ins Bewußtsein. Auch im Produktionsbereich kämpfen die Gewerkschaften nicht mehr nur für höhere Bezahlung, sondern richten sich zunehmend gegen den Umfang der Arbeitszeit, gegen die Formen der Arbeitskontrolle und Betriebshierarchie, gegen Rationalisierungstechnologie und damit verbundene Arbeitsformen, kurz, gegen die kapitalistische Form des Arbeitsprozesses samt den in sie eingeschlossenen subjektiven Folgen. Wie widersprüchlich, beschränkt unzusammenhängend und auf unterschiedlicher materieller Basis stattfindend diese $\mathrm{Be}$ wegungen auch sein mögen, sie sind doch die Grundlage dafür, daß viele marxistische Traditionen, die die sozialistische Entfesselung der Produktivkräfte propagierten, problematisch wurden. Marx war hier ungebrochener Anhänger des Fortschrittsglaubens des 19. Jahrhunderts, auch wenn bei ihm viel klarer als bei allen folgenden Marxisten die kapitalistische Formung des Arbeitsprozesses, der Fabrik und des Verhältnisses von Natur und Mensch angesprochen war. Darin war in abstracto die Möglichkeit einer anderen Formung des Produktionsprozesses enthalten, aber nicht als konkretes Projekt einer Alternative. Der Marxismus der 2. Internationale verlän- 
gerte die kapitalistische Technologie problemlos in die Zukunft, und ebenso unproblematisch war dem Marxismus der 3. Inte mationale die staatliche Industrialisierung auf der Grundlage der importierten und imitierten kapitalistischen Produktivkräfte. Der Marxismus der Neuen Linken konnte dem wenig hinzufügen, wenn er auch auf dem Hintergrund der chinesischen Kulturrevolution den Topos einer neuen Technik wenigstens wieder denken konnte. Inzwischen reicht diese Naivität nicht mehr hin. Sowohl die Agonie des kapitalistischen Industrialismus in den entwickelten Zentren, wie auch dessen eurozentrische Verlängerung als Basis der Entwicklung der Unterentwicklung verlangen neue Produktions- und Reproduktionsformen auf nationaler wie internationaler Ebene. Nicht im Sinne eines romantischen Antiindustrialismus, sondern im Sinne eines emanzipatorischen Industrialismus wird der Marxismus wenigstens eine ganz neue Offenheit entwickeln müssen.

\section{Objektivismus}

Ökonomismus, Etatismus und Industrialismus als Brennpunkte der Krise des Marxismus stellen nicht einfach falsches Begreifen der Entwicklungsrealität kapitalistischer Gesellschaftsverhältnisse dar, sondern in Arbeiterbewegungen eingelassene Interpretations- und Orientierungsmuster, die historisch ihre Unzulänglichkeit erfahren mußten und dies heute anläßlich der realen Entwicklungsdynamik sozialer Bewegungen erneut erfahren müssen. Damit werden bestimmte Seiten der Marxschen Theorie wiederentdeckt, geschichtlich unwirksame Marxismen wieder in ihrer kritischen Potenz aktuell und vor allem ganz neue Dimensionen geschichtsemanzipatorischen Denkens akut. Die Krise des Marxismus in ihren unterschiedlichen Dimensionen läßt sich generell als Problem des Verhältnisses von gesellschaftlichem Emanzipationsprozeß und Marxismus zusammenfassen. Der Marxismus unterliegt dabei prinzipiell der Gefahr des Objektivismus, d.h. bestimmte Entwicklungsformen in der Geschichte des Kapitalismus und bestimmte Konstitutionsformen des Klassenkampfs und der sozialen Bewegungen werden historisch hypostasiert und zum geschichtsnotwendigen Entwicklungsgang der Emanzipation stilisiert, also verdinglicht. Dem korrespondiert eine Partei von marxistisch ,Wissenden', die theoretisch vorherbestimmten Klassen und Schichten für diesen Entwicklungsprozeß zu mobilisieren, zu organisieren und aufzuklären haben. Die Geschichte der Spaltungen der Arbeiterbewegungen und der Ausschließung alternativer marxistischer Strömungen, die Frage der Bündnispolitik als machtpolitischem Kalkül und die Abdrängung anderer sozialer Bewegungen wie etwa der Frauenbewegung als sekundär - alle diese marxistischen Traditionen sind angesichts der Dynamik der neuen sozialen Bewegungen, die in weiten Teilen am Marxismus vorbellaufen, nur zu offenkundig problematisch. Keineswegs geht es dabei um ein opportunistisches Nachlaufen hinter diesen Bewegungen, aber der Marxismus muß sich in der Erklärung und Kritik gesellschaftlicher Entwicklungen inhaltlich auf die Handlungsprozesse einlassen müssen, denn nur dann wird er aufklären, orientieren und vor allem selbst lernen können. Der Geschichtsobjektivismus des Marxismus hat nur allzuoft zum Modeln 
der historischen Handlungsprozesse nach den eigenen Vorstellungen geführt und entsprechend zur Negation der theoretischen: „bürgerlichen“, alltäglichen und wissenschaftlichen Bewußtseinsformen, in denen sich das Handeln der Menschen vollzieht - Marxismus als ,Ausgrenzungsgeschichte" $(\mathrm{O}$. Negt). In der Krise des Marxismus geht es um die selbstkritische Frage: Welche theoretische und praktische Bedeutung hatte der Marxismus im gesellschaftlichen Emanzipationsprozeß und welche Bedeutung wird er zukünftig haben?

Redaktion,Prokla

Heft Nr. 27 der PROKLA erscheint im Oktober 1979 mit den Schwerpunkten "Europäische Integration - Altemativen der Wirtschaftspolitik" mit Beiträgen von E. Altvater, K. Busch, C. Leggewie, R. Hickel, M. Funke u. a.

Heft Nr. 38 der PROKLA erscheint im Dezember 1979 mit dem Schwerpunkt "Weltmarkt und Unterentwicklung" 